\title{
Modulation of intracellular iron metabolism by iron chelation affects chromatin remodeling proteins and corresponding epigenetic modifications in breast cancer cells and increases their sensitivity to chemotherapeutic agents
}

\author{
IGOR P. POGRIBNY, VOLODYMYR P. TRYNDYAK, MARTA POGRIBNA, SVITLANA SHPYLEVA, \\ GORDON SURRATT, GONÇALO GAMBOA DA COSTA and FREDERICK A. BELAND
}

Division of Biochemical Toxicology, National Center for Toxicological Research, FDA, Jefferson, AR 72079, USA

Received November 29, 2012; Accepted January 30, 2013

DOI: $10.3892 /$ ijo.2013.1855

\begin{abstract}
Iron plays a vital role in the normal functioning of cells via the regulation of essential cellular metabolic reactions, including several DNA and histone-modifying proteins. The metabolic status of iron and the regulation of epigenetic mechanisms are well-balanced and tightly controlled in normal cells; however, in cancer cells these processes are profoundly disturbed. Cancer-related abnormalities in iron metabolism have been corrected through the use of iron-chelating agents, which cause an inhibition of DNA synthesis, $G_{1}-S$ phase arrest, an inhibition of epithelial-to-mesenchymal transition, and the activation of apoptosis. In the present study, we show that, in addition to these well-studied molecular mechanisms, the treatment of wild-type TP53 MCF-7 and mutant TP53 MDA-MB-231 human breast cancer cells with desferrioxamine (DFO), a model iron chelator, causes significant epigenetic alterations at the global and gene-specific levels Specifically, DFO treatment decreased the protein levels of the
\end{abstract}

Correspondence to: Dr Igor P. Pogribny, Division of Biochemical Toxicology, National Center for Toxicological Research (NCTR), 3900 NCTR Rd., Jefferson, AR 72079, USA

E-mail: igor.pogribny@fda.hhs.gov

Abbreviations: DFO, desferrioxamine; JHDM, Jumonji domaincontaining histone demethylase; TET, TET methylcytosine dioxygenase; $\mathrm{p} 21$, cyclin-dependent kinase inhibitor 1A; HIF1 $\alpha$, hypoxiainducible factor $1 \alpha$; H3K9me3, trimethyl histone $\mathrm{H} 3$ lysine 9; H3K9me2, dimethyl histone H3 lysine 9; H3K9ac, acetyl histone H3 lysine 9; H3K27me3; trimethyl histone H3 lysine 27; H3K36me3, trimethyl histone $\mathrm{H} 3$ lysine 36 ; $\mathrm{H} 3 \mathrm{~K} 4 \mathrm{me} 3$, trimethyl histone $\mathrm{H} 3$ lysine 4; H3K4me2, dimethyl histone H3 lysine 4; H4K20me3, trimethyl histone H4 lysine 20; LC-MS/MS, liquid chromatographytandem mass spectrometry; ChIP, chromatin immunoprecipitation; DOX, doxorubicin hydrochloride; CDDP, cis-diammineplatinum(II) dichloride; HMT, histone methyltransferase

Key words: breast cancer, iron metabolism, histone modifications, DNA methylation histone $\mathrm{H} 3$ lysine 9 demethylase, Jumonji domain-containing protein 2A (JMJD2A), in the MCF-7 and MDA-MB-231 cells and down-regulated the levels of the histone $\mathrm{H} 3$ lysine 4 demethylase, lysine-specific demethylase 1 (LSD1), in the MDA-MB-231 cells. These changes were accompanied by alterations in corresponding metabolically sensitive histone marks. Additionally, we demonstrate that DFO treatment activates apoptotic programs in MCF-7 and MDA-MB-231 cancer cells and enhances their sensitivity to the chemotherapeutic agents, doxorubicin and cisplatin; however, the mechanisms underlying this activation differ. The induction of apoptosis in wild-type TP53 MCF-7 cells was p53-dependent, triggered mainly by the down-regulation of the JMJD2A histone demethylase, while in mutant TP53 MDA-MB-231 cells, the activation of the p53-independent apoptotic program was driven predominantly by the epigenetic up-regulation of $\mathrm{p} 21$.

\section{Introduction}

A growing body of evidence indicates the existence of an intimate link between the metabolic status and epigenetic regulation of cells $(1,2)$. This is exemplified by the fact that a variety of small molecules involved in intercellular metabolism, including adenosine triphosphate, $\mathrm{S}$-adenosylmethionine, nicotinamide adenine dinucleotide, flavin adenine dinucleotide, folate, acetyl coenzyme A, $\alpha$-ketoglutarate and iron, are essential for the proper maintenance of the cellular epigenome.

Iron is an essential trace element for normal cellular function. In addition to its significance in controlling a variety of cellular processes, including proliferation, DNA synthesis and repair, and mitochondrial electron transport, which are essential for the accurate maintenance of normal cellular homeostasis, iron plays a key regulatory role in the functioning of DNA and histone-modifying proteins $(3,4)$. Specifically, the Jumonji domain-containing histone demethylase (JHDM) family, which catalyzes the demethylation of tri- and dimethylated lysine 9 and lysine 36 residues in histone $\mathrm{H} 3(5,6)$ and ten-eleven translocation 1-3 (TET1-3; TET methylcytosine dioxygenase 1-3), proteins that catalyze the hydroxylation of 5-methylcytosine to form 5-hydroxymethylcytosine (7), are 
members of the superfamily of $\alpha$-ketoglutarate-non-heme $\mathrm{Fe}^{+2}$-dependent oxygenases (8). This provides a direct link between the regulation of epigenetic mechanisms and the status of cellular iron metabolism. Both of these processes are well-balanced and tightly controlled in normal cells; however, in cancer cells these processes are profoundly disturbed $(9,10)$. For instance, it is well-established that changes in cellular iron metabolism play a crucial role in the progression of many types of cancer (9), including breast cancer (11), suggesting that deregulated iron metabolism in malignant cells may be a promising molecular target for cancer therapy. Similarly, the dysregulation of epigenetic mechanisms is regarded as one of the hallmarks of cancer $(12,13)$ and correcting the enzymatic processes that control the epigenome has emerged as a novel epigenetic approach for the treatment of cancer (13).

It has previously been reported that agents that modulate iron metabolism in cancer cells, particularly those that restrict iron availability, including iron chelating agents $(14,15)$ and transferrin receptor-targeted treatments (16), exhibit potent and broad anti-tumor activity. Several studies have demonstrated that the mechanism of the anti-tumor action of iron chelators is linked to the inhibition of DNA synthesis, the induction of DNA damage, $G_{1}-S$ phase cell cycle arrest, and the activation of apoptosis $(15,17)$; however, research on targeting iron metabolism in cancer cells for anti-tumor therapy is still in its infancy (15).

Based on these considerations, we hypothesized that, in addition to the well-studied molecular mechanisms for the action of iron chelators, their anti-tumor activity may be associated with modulating the function of chromatin-modifying proteins and their corresponding metabolically sensitive epigenetic modifications in cancer cells. The results of the present study demonstrate that the treatment of wild-type TP53 MCF-7 and mutant TP53 MDA-MB-231 human breast cancer cells with desferrioxamine (DFO), a model iron chelator, causes significant epigenetic alterations at the global and gene-specific levels and that these alterations activate cellular apoptotic programs and enhance the sensitivity of cancer cells to the chemotherapeutic agents, doxorubicin and cisplatin.

\section{Materials and methods}

Cell lines, cell culture and treatment. MCF-7 (wild-type TP53) and MDA-MB-231 (mutant TP53) human breast cancer cell lines were obtained from the American Type Culture Collection (ATCC, Manassas, VA) and maintained according to the manufacturer's recommendations. DFO mesylate (DFO) was purchased from Sigma-Aldrich (St. Louis, MO). In the experiments with DFO, cells were seeded at a density of $0.5 \times 10^{6}$ viable cells per $100-\mathrm{mm}$ plate. Twenty-four hours after seeding, the medium was changed and fresh medium containing $100 \mu \mathrm{M}$ of DFO and supplemented with $10 \%$ fetal bovine serum was added. At 6, 24 and $48 \mathrm{~h}$ after the addition of DFO, the cells were scraped on ice, washed in phosphate-buffered saline, and immediately frozen at $-80^{\circ} \mathrm{C}$ for subsequent analyses.

Western blot analysis of proteins. Whole cell lysates were prepared by homogenization in $200 \mu \mathrm{l}$ of lysis buffer $(50 \mathrm{mM}$ Tris- $\mathrm{HCl}, \mathrm{pH} 7.4 ; 1 \%$ NP-40; $0.25 \%$ sodium deoxycholate; $150 \mathrm{mM} \mathrm{NaCl} ; 1 \mathrm{mM}$ EDTA; $1 \mathrm{mM}$ PMSF; $1 \mu \mathrm{g} / \mathrm{ml}$ each of aprotinin, leupeptin and pepstatin; $1 \mathrm{mM} \mathrm{Na}_{3} \mathrm{VO}_{4}$, and $1 \mathrm{mM}$
$\mathrm{NaF}$ ), sonication and incubation at $4^{\circ} \mathrm{C}$ for $30 \mathrm{~min}$, followed by centrifugation at $12,000 \mathrm{x}$ g at $4^{\circ} \mathrm{C}$ for $10 \mathrm{~min}$. Extracts containing equal quantities of proteins were separated by SDS-PAGE on $8 \%, 10 \%$, or $15 \%$ polyacrylamide gels and transferred onto PVDF membranes. The membranes were probed with primary antibodies against Jumonji domaincontaining protein 2A (JMJD2A; 1:1,000; Sigma-Aldrich), TET2 (1:1,000; Abcam, Cambridge, MA), histone demethylase SWIRM1 (lysine-specific demethylase LSD1; 1:1,000; Sigma-Aldrich), cyclin-dependent kinase inhibitor 1A (p21; Cip1; Cell Signaling Technology, Danvers, MA), p53 (1:1,000; Cell Signaling Technology) and hypoxia-inducible factor $1 \alpha$ (HIF1 $\alpha$; 1:200; Santa Cruz Biotechnology, Santa Cruz, CA). Alkaline phosphatase-conjugated secondary antibodies (EMD Millipore, Billerica, MA) were used for visualization. Equal protein loading was confirmed by immunostaining against $\beta$-actin (1:4,000; Sigma-Aldrich). The signal intensity was analyzed using ImageQuant software (Molecular Dynamics, Sunnyvale, CA) and normalized to $\beta$-actin.

Jumonji-type and LSD-type histone demethylase activity assay. The activity of Jumonji-type and LSD-type histone demethylases was determined using Demethylase Activity Assay kits (Cayman Chemical Co., Ann Arbor, MI) according to the manufacturer's instructions.

Western blot analysis of histone modifications. The histone modification status in the untreated MCF-7 and MDA-MB-231 cells and cells treated with DFO was determined by western blot analysis using the following primary antibodies: anti-trimethyl histone H3 lysine 9 (H3K9me3; 1:1,000), anti-dimethyl histone $\mathrm{H} 3$ lysine 9 (H3K9me2; 1:1,000), anti-acetyl histone $\mathrm{H} 3$ lysine 9 (H3K9ac; 1:1,000), anti-trimethyl histone $\mathrm{H} 3$ lysine 27 (H3K27me3; 1:1,000), anti-trimethyl histone H3 lysine 36 (H3K36me3; 1:1,000), anti-trimethyl histone H3lysine 4 (H3K4me3; 1:1,000), anti-dimethyl histone $\mathrm{H} 3$ lysine 4 (H3K4me2; 1:1,000) and anti-trimethyl histone $\mathrm{H} 4$ lysine 20 (H4K20me3; 1:1,000) as previously described (18). All antibodies were obtained from EMD Millipore.

Quantitative reverse transcription real-time PCR (qRT-PCR). Total RNA was extracted from the breast cancer cell lines using TRI Reagent (Ambion, Austin, TX) according to the manufacturer's instructions. Reverse transcription was performed using High Capacity cDNA Reverse Transcription kits (Applied Biosystems, Foster City, CA) and cDNA was analyzed in a 96-well plate assay format using a 7900HT Fast Real-Time PCR System (Applied Biosystems). Each plate contained one experimental gene and one housekeeping gene. All primers were obtained from Applied Biosystems. The relative mRNA level for each gene was determined using the $2^{-\Delta \Delta \mathrm{Ct}}$ method (19). The results are presented as a fold change for each mRNA in the DFO-treated cells relative to the untreated control cells.

Determination of global DNA methylation. The extent of global DNA methylation was determined by slight modification of the liquid chromatography-tandem mass spectrometry (LC-MS/MS) method (20). The analyses were conducted in a system comprising a Waters Acquity UltraPerformance Liquid Chromatograph (UPLC; Waters Corporation, Milford, MA), 
coupled with a Waters Quattro Premier XE triple quadrupole mass spectrometer operating in positive ion electrospray mode. DNA samples $(2 \mu \mathrm{g})$ dissolved in $20 \mu \mathrm{l}$ of Tris-EDTA buffer, $\mathrm{pH} 8.0$, were added to $180 \mu \mathrm{l}$ of formic acid $(98 \%$, Sigma-Aldrich) and to each sample were added $275 \mathrm{ng}$ of cytosine-2,4-1 ${ }^{13} \mathrm{C}_{2},{ }^{15} \mathrm{~N}_{3}$ (Sigma-Aldrich) and $20 \mathrm{ng}$ of 5-methyl$d_{3}$-cytosine-6- $d_{1}$ (5-MeC- $d_{4} ; \mathrm{C} / \mathrm{D} / \mathrm{N}$ Isotopes, Pointe-Claire, $\mathrm{QC}$, Canada). The mixtures were sealed in 2-ml autosampler vials and incubated in a thermostated heating block at $140^{\circ} \mathrm{C}$ for $90 \mathrm{~min}$. After cooling to the room temperature the vials were opened, dried in a centrifugal evaporator, and the contents were dissolved in $1 \mathrm{ml}$ of $95 \%$ acetonitrile/5\% water in a sonicator bath for $15 \mathrm{~min}$. Each sample $(5 \mu \mathrm{l})$ was injected in the UPLC system and eluted in a Waters UPLC BEH HILIC column $(1.7 \mu \mathrm{m}, 2.1 \mathrm{~mm} \times 100 \mathrm{~mm})$ using an isocratic elution with $93 \%$ of acetonitrile and $7 \%$ of $2.5 \mathrm{mM}$ ammonium formate at $200 \mu \mathrm{l} / \mathrm{min}$. Under these chromatographic conditions, cytosine eluted at $\mathrm{ca} .4 .75 \mathrm{~min}$ and 5-methylcytosine (5-mC) eluted at ca. $5.49 \mathrm{~min}$. The mass spectral acquisition was conducted using multiple reaction monitoring as follows: cytosine, $\mathrm{m} / \mathrm{z} \quad 112.1 \rightarrow 95.1 ;{ }^{13} \mathrm{C}^{15} \mathrm{~N}$-cytosine, $\mathrm{m} / \mathrm{z} \quad 117.1 \rightarrow 72.2$; $5-\mathrm{mC}, \mathrm{m} / \mathrm{z} 126.1 \rightarrow 109.1$; and $5-\mathrm{mC}_{-} d_{4}, \mathrm{~m} / \mathrm{z} 130.2 \rightarrow 113.2$. A plot of the response ratio for labeled versus unlabeled cytosine was linear $\left(r^{2}>0.999\right)$ over a concentration range of $0-700 \mathrm{ng} / \mathrm{ml}$ cytosine with $275 \mathrm{ng} / \mathrm{ml}^{13} \mathrm{C}^{15} \mathrm{~N}$-cytosine. A plot of the response ratio for labeled versus unlabeled $5-\mathrm{mC}$ was linear $\left(\mathrm{r}^{2}>0.999\right)$ over a concentration range of $0-80 \mathrm{ng} / \mathrm{ml} 5-\mathrm{mC}$ with $20 \mathrm{ng} / \mathrm{ml}$ $5-\mathrm{mC}-d_{4}$. The percentage of methylation was calculated as the quotient between the number of moles of 5-mC and the sum of the number of moles of 5-mC and cytosine in each sample. The methodology was validated as regards its accuracy and precision by analyzing, on 2 consecutive days, 5 samples of salmon testes DNA (Sigma-Aldrich) and 5 samples of salmon testes DNA spiked with 5-mC. The intra- and inter-day imprecision of the method, assessed by the relative standard deviation of the replicate analyses conducted on each day was under $2.1 \%$. The intra- and inter-day inaccuracy of the method assessed in the analysis of the spiked versus unspiked samples ranged from $0.4-2.0 \%$.

Determination of $\mathrm{Cp} G$ island methylation status by cytosine extension assay. The status of $\mathrm{CpG}$ island methylation evaluated with a radiolabeled $\left[{ }^{3} \mathrm{H}\right] \mathrm{dCTP}$ extension assay (21) following digestion of DNA with methylation-sensitive TspMI restriction endonuclease, whose CCCGGG hexanucleotide recognition sequences occur predominantly within $\mathrm{CpG}$ islands.

Chromatin immunoprecipitation assay. Formaldehyde crosslinking and chromatin immunoprecipitation (ChIP) assays were performed with primary antibodies against histone H3K9me2 (EMD Millipore) and H3K4me2 (EMD Millipore) using a MAGnify Chromatin Immunoprecipitation System (Invitrogen, Carlsbad, CA). Purified DNA from the immunoprecipitates and from input DNA was analyzed by quantitative real-time PCR (qPCR) on an Applied Biosystems 7900HT Fast Real-Time PCR System using the following primer set: 5'-GTGGCTCTGATTGGCTTTCTG-3' (forward) and 5'-CCA GCCCTGTCGCAAGGATC-3' (reverse) for the promoter of the human $p 21$ gene (22). The results were normalized to the amount of input DNA and presented as the fold change in the amount of immunoprecipitated DNA isolated from the DFO-treated cells relative to the untreated control cells.

Drug sensitivity assay. To assay drug sensitivity, MCF-7 and MDA-MB-231 cells were plated at a density of $5 \times 10^{3}$ cells per well in 96-well plates. Cells were cultured in medium containing $100 \mu \mathrm{M}$ of DFO. After $48 \mathrm{~h}$ of incubation, the medium was changed, and the cells were treated with doxorubicin hydrochloride (DOX) or cis-diammineplatinum(II) dichloride (CDDP) purchased from Sigma-Aldrich. Cell survival was analyzed with a CellTiter-Blue ${ }^{\circledR}$ Cell Viability assay (Promega, Madison, WI). The $\mathrm{IC}_{50}$ (inhibitory concentration to produce $50 \%$ cell death) values were determined using the resulting dose-response curves. The experiments were repeated twice, and each cell line examined in triplicate.

Statistical analyses. The results are presented as the means \pm SD. Statistical analyses were conducted by one-way analysis of variance, with pair-wise comparisons conducted using the Student-Newman-Keuls test.

\section{Results}

DFO affects the protein level of histone demethylases. Fig. 1 demonstrates that the protein level of JMJD2A was significantly decreased in the MCF-7 and MDA-MB-231 cells cultured for 24 and $48 \mathrm{~h}$ in medium containing DFO, with the changes being more pronounced in the DFO-treated MCF-7 cells. This was evident by the fact that the level of JMJD2A protein in the MCF-7 cells was decreased by 65 and $66 \%$ at 24 and $48 \mathrm{~h}$, respectively after the initiation of DFO treatment, whereas the protein level of JMJD2A in the DFO-treated MDA-MB-231 cells was reduced only by 31 and $39 \%$, respectively.

Culturing MDA-MB-231 cells in the DFO-containing medium for 24 and $48 \mathrm{~h}$ resulted in a significant reduction in LSD1 protein levels by 63 and 40\%, respectively. By contrast, the protein level of LSD1 in the DFO-treated MCF-7 cells did not differ from the values in the MCF-7 untreated control cells, apart from a slight transient increase observed after $24 \mathrm{~h}$ of DFO treatment. The respective changes in the protein levels of JMJD2A and LSD1 in the DFO-treated MCF-7 and MDA-MB-231 cells were accompanied by decreases in their enzymatic activity (data not shown).

The levels of TET2 protein in the MCF-7 and MDA-MB-231 cells cultured in DFO-containing medium decreased, with the values being significant at 24 and 48 h; however, the reduction in TET2 protein levels in the DFO-treated MDA-MB-231 cells was more pronounced compared to the MCF-7 cells at the same time points (Fig. 1).

DFO affects the extent of global histone modification pattern. The down-regulation of JMJD2A in the DFO-treated MCF-7 cells and JMJD2A and LSD1 in the DFO-treated MDA-MB-231 cells was accompanied by changes in the triand dimethylation of histone $\mathrm{H} 3 \mathrm{~K} 9$, the acetylation of histone $\mathrm{H} 3 \mathrm{~K}$ 9, the trimethylation of histone H3K36 and the dimethylation of histone H3K4 (Fig. 2). Specifically, the level of histone H3K9me3 was slightly although significantly increased in the DFO-treated MDA-MB-231 cells at 6, 24 and $48 \mathrm{~h}$, while in the MCF-7 cells only at $48 \mathrm{~h}$. 

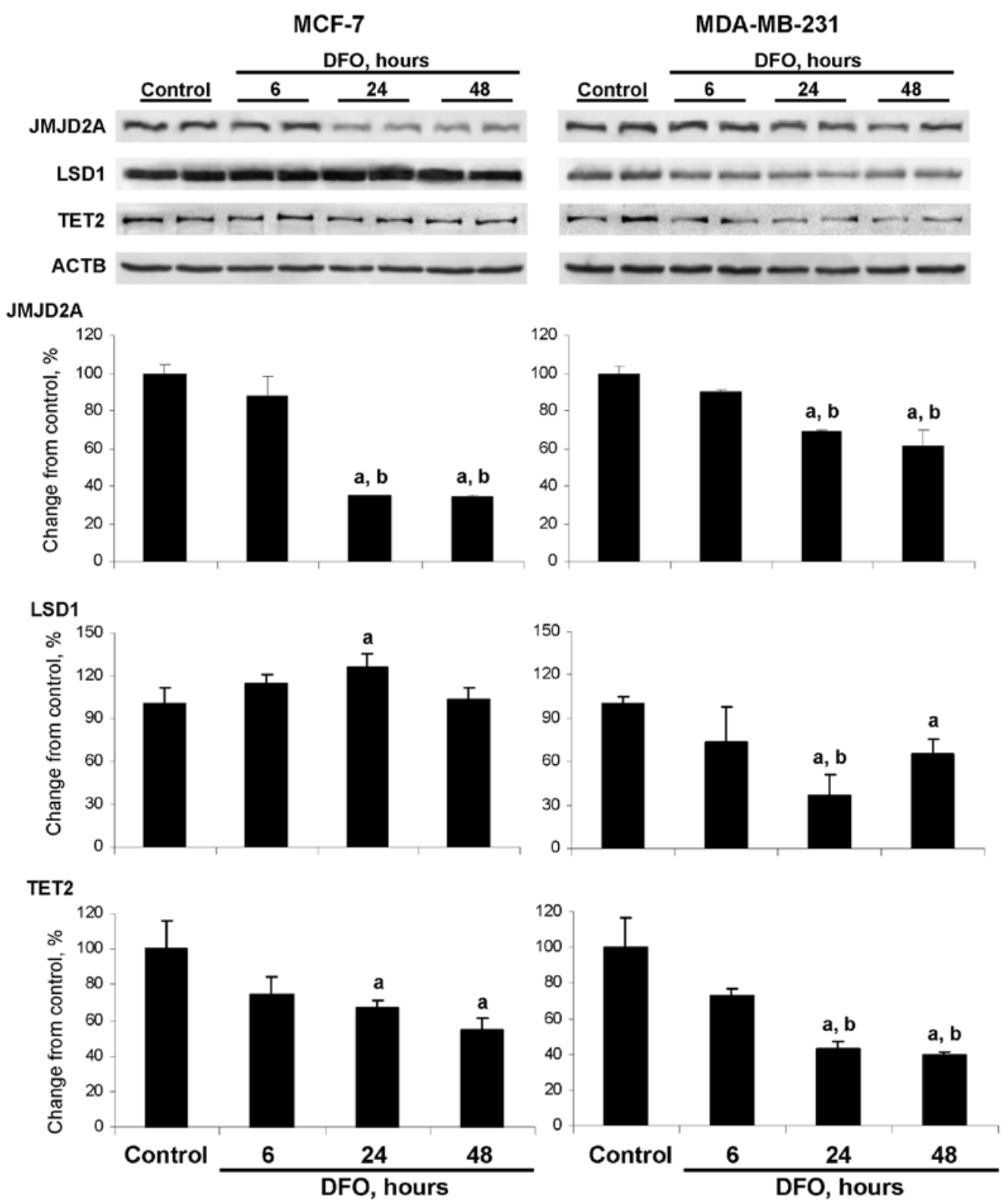

Figure 1. Western blot analysis of JMJD2A, LSD1 and TET2 proteins in the untreated and DFO-treated MCF-7 and MDA-MB-231 human breast cancer cells. MCF-7 and MDA-MB-231 cells were cultured in medium containing $100 \mu \mathrm{M}$ of DFO and supplemented with $10 \%$ fetal bovine serum for 6, 24, or 48 h. Cell lysates were separated by SDS-PAGE and subjected to western immunoblot analysis using specific antibodies against JMJD2A, LSD1 and TET2 proteins. Equal sample loading was confirmed by immunostaining against $\beta$-actin (ACTB). Representative western immunoblot images are shown. Data are presented as the percentage change from the corresponding untreated control cells, which were assigned a value of $100 \%$. The letters ' $a$ ' and ' $b$ ' denote a significant $(\mathrm{p}<0.05)$ difference $(\mathrm{n}=3)$ compared to the corresponding untreated control cells (a), or the cells treated with DFO for $6 \mathrm{~h}$ (b). These results were reproduced in 2 independent experiments.

Culturing the MCF-7 and MDA-MB-231 cells in DFOcontaining medium resulted in an increase in histone $\mathrm{H} 3 \mathrm{~K} 9 \mathrm{me} 2$ levels, particularly in MCF-7 cells. This was evident by a marked time-dependent elevation of histone H3K9me2 in the DFO-treated MCF-7 cells, with the highest values observed at $48 \mathrm{~h}$. At this time point, the level of histone $\mathrm{H} 3 \mathrm{~K} 9 \mathrm{me} 2$ was $254 \%$ greater than in the untreated MCF-7 control cells and significantly greater than the values at 6 and $24 \mathrm{~h}$ in the DFO-treated MCF-7 cells (Fig. 2). In the DFO-treated MDA-MB-231 cells, the levels of histone $\mathrm{H} 3 \mathrm{~K} 4 \mathrm{me} 2$ were only slightly increased compared to the untreated MDA-MB-231 control cells and were relatively constant at each time point.

The levels of histone H3K9ac were decreased in the DFO-treated MCF-7 and MDA-MB-231 cells at 24 and $48 \mathrm{~h}$; however, the extent of histone $\mathrm{H} 3 \mathrm{~K} 9 \mathrm{ac}$ reduction was greater in the MCF-7 cells. This corresponds to the well-established inverse association between histone H3K9 methylation and histone H3K9 acetylation (23).

The pattern of changes in the levels of histone H3K36me3 in the DFO-treated MCF-7 cells was similar to the alterations in the levels of histone H3K9me2 in these cells, which may be attributed to the fact that JMJD2A is responsible for the demethylation of both of these histone modification markers $(5,6)$. By contrast, the level of histone H3K36me3 in the DFO-treated MDA-MB-231 cells did not differ from the values in the untreated cells.

Culturing the MCF-7 and MDA-MB-231 cells in DFO-containing medium resulted in an increase in histone 


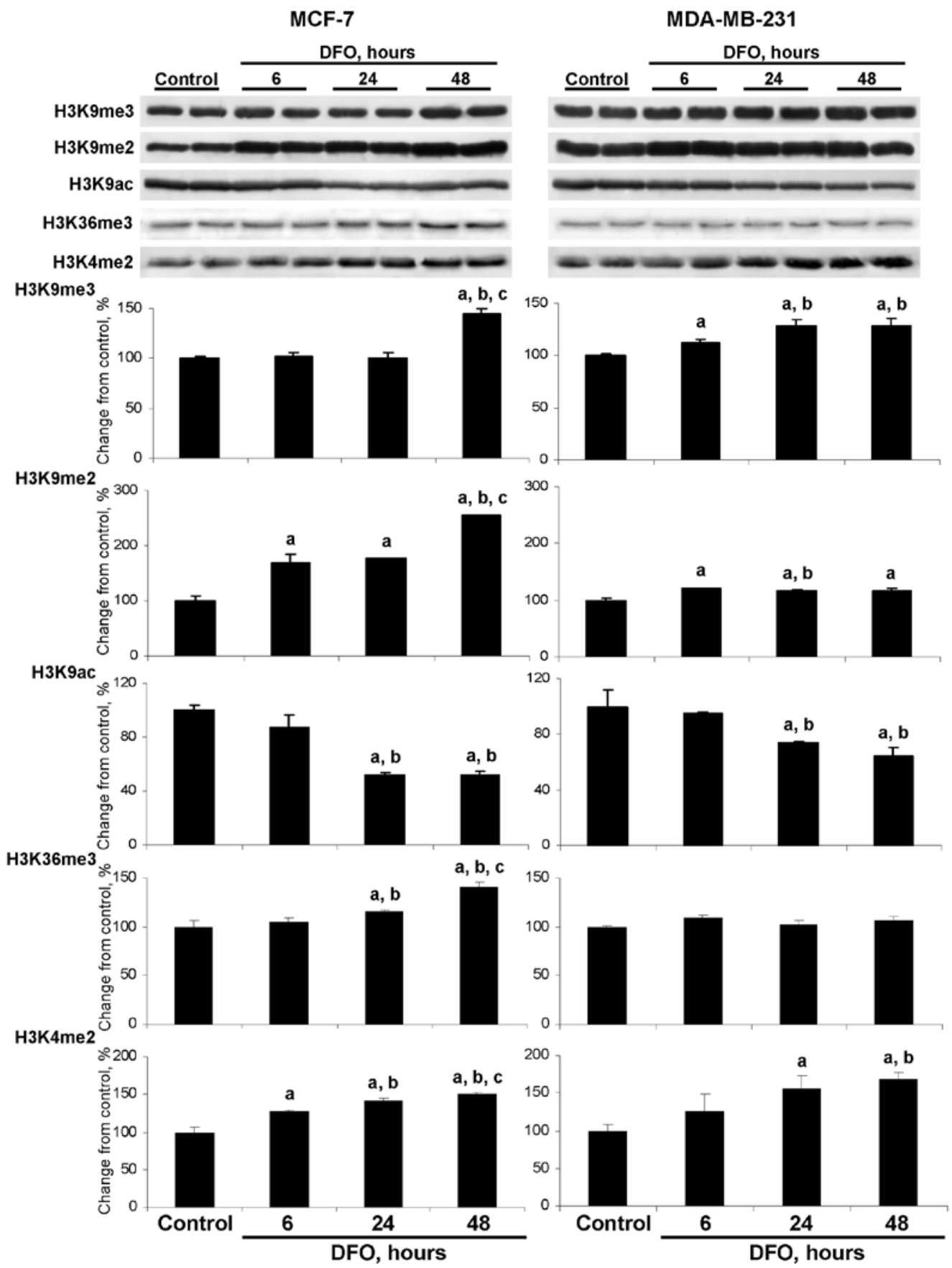

Figure 2. Effects of DFO treatment on histone H3 lysine modifications in MCF-7 and MDA-MB-231 human breast cancer cells. Histone H3K9me3, H3K9me2, H3K9ac, H3K36me3 and H3K4me2 levels were assessed by immunostaining using specific antibodies against these modifications. Densitometry analysis of the immunostaining results is shown as the percentage change in histone modification levels in the DFO-treated MCF-7 and MDA-MB-231 cells relative to the values in the corresponding untreated control cells. The letters ' $a$ ', ' $b$ ' and ' $c$ ' denote a significant $(p<0.05)$ difference $(n=3)$ from the corresponding untreated control cells (a), or the cells treated with DFO for 6 (b) or $24 \mathrm{~h}$ (c).

H3K4me2 levels in both cell lines. By contrast, the levels of histone $\mathrm{H} 3 \mathrm{~K} 4 \mathrm{me} 3$ did not change (data not shown), which may be explained by the fact that LSD1 demethylates only dimethylated histone $\mathrm{H} 3$ lysine 4 residues (24).

Additionally, the levels of histone $\mathrm{H} 3 \mathrm{~K} 27 \mathrm{me} 3$ and histone H4K20me3 in the DFO-treated MCF-7 and MDA-MB-231 cells did not differ from the values in the respective untreated cells (data not shown).

Effect of DFO on DNA and the expression of histone-modifying genes. To further evaluate the effect of DFO treatment on the functioning of DNA and histone methylation machinery, qRT-PCR was conducted to examine the expression of histone methyltransferases (HMTs), including the histone H3K9 methyltransferases, $S U V 39 H 1$ and EHMT2, the histone H3K4 methyltransferases, SET1 and ASH2, and the histone H3K36 methyltransferase SETD2, as well as that of the DNA methyltransferases (DNMTs), DNMT1, DNMT3A and DNMT3B.

Fig. 3 demonstrates that culturing the MCF-7 and MDA-MB-231 cells in DFO-containing medium resulted in cell type-dependent alterations in the expression of HMT genes. This was evident by a difference in the trends and magnitude 

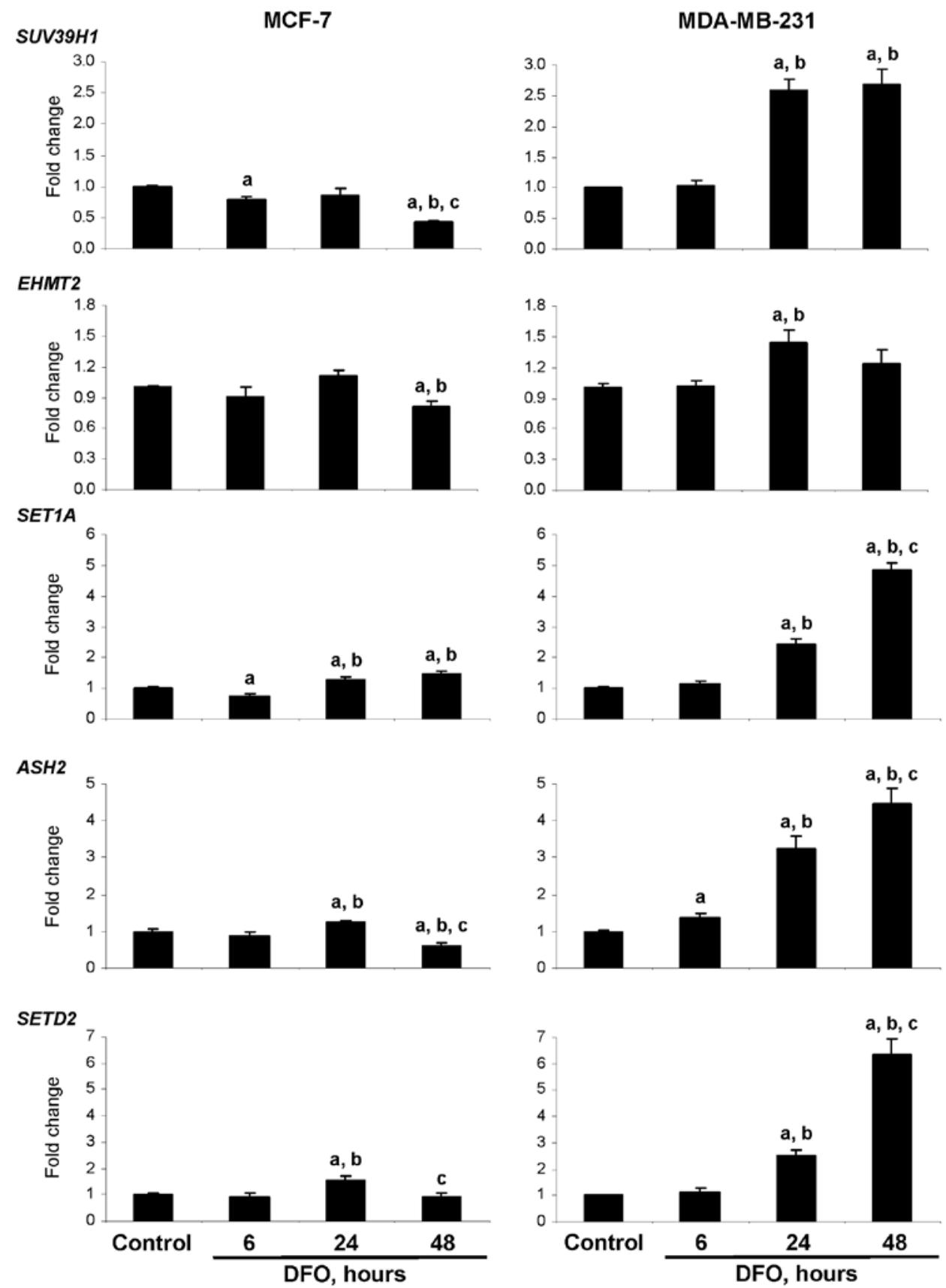

Figure 3. Expression of the SUV39H1, EHMT2, SET1A, ASH2 and SETD2 histone methyltransferase genes in the untreated and DFO-treated MCF-7 and MDA-MB-231 human breast cancer cells. Total RNA from the untreated and DFO-treated cells was used to evaluate the transcriptional abundance of SUV39H1, EHMT2, SET1A, ASH2 and SETD2 genes. The gene expression was determined by qRT-PCR as detailed in 'Materials and methods'. Data are presented as an average fold change in the expression of each gene in the DFO-treated cells relative to that in the corresponding untreated control cells, which were assigned the value 1 . The letters ' $a$ ', ' $b$ ' and ' $c$ ' denote a significant $(\mathrm{p}<0.05)$ difference $(n=3)$ compared to the corresponding untreated control cells $(a)$, or the cells treated with DFO for 6 (b) or $24 \mathrm{~h}(\mathrm{c})$.

of the expression changes in the DFO-treated MCF-7 and MDA-MB-231 cells. The most noticeable changes were a distinct up-regulation in the expression of the SUV39H1,SETIA, $A S H 2$ and SETD2 HMTs in the DFO-treated MDA-MB-231 cells at $24 \mathrm{~h}$ and, specifically, at $48 \mathrm{~h}$. By contrast, the expression of the SUV39H1, EHMT2, ASH2 and SETD2 HMTs in the DFO-treated MCF-7 cells was significantly lower at $48 \mathrm{~h}$. Similar to the HMTs, the expression of DNMT1, DNMT3A and $D N M T 3 B$ in the MDA-MB-231 cells cultured in the presence of DFO was markedly increased at 24 and $48 \mathrm{~h}$ with the magnitude being greater at $48 \mathrm{~h}$ (Fig. 4A). By contrast, the expression of DNMTs in the DFO-treated MCF-7 cells was decreased, reaching the lowest level $48 \mathrm{~h}$ after the initiation of DFO treatment. At this time, the expression of DNMT1, DNMT3A and DNMT3B in the DFO-treated MCF-7 cells was 75, 65 and $72 \%$ lower, respectively, as compared to similar values in the untreated MCF-7 control cells.

Effect of DFO on DNA methylation. Fig. 4B demonstrates that culturing the MCF-7 cells in DFO-containing medium did not affect the level of 5-mC in the DNA. By contrast, the level of 5-mC in the DFO-treated MDA-MB-231 cells was reduced by 
A

DNMT1

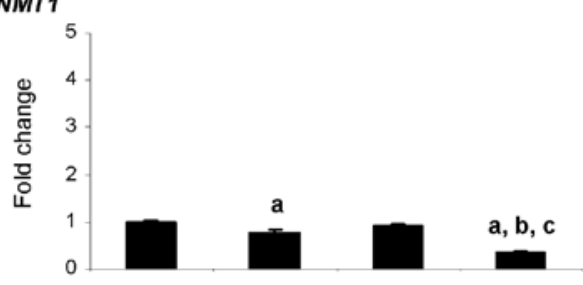

DNMT 3 A

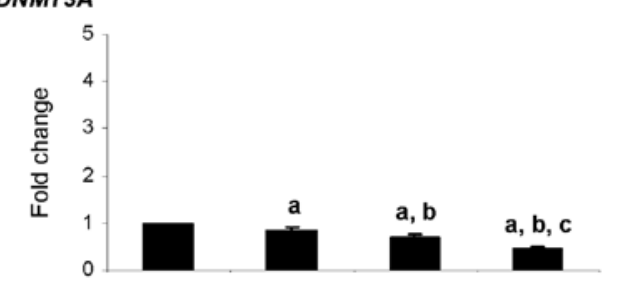

DNMT3B

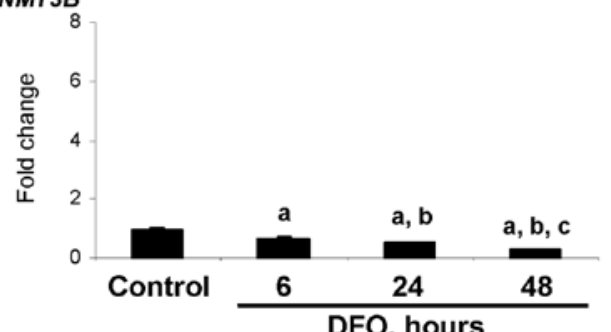

B

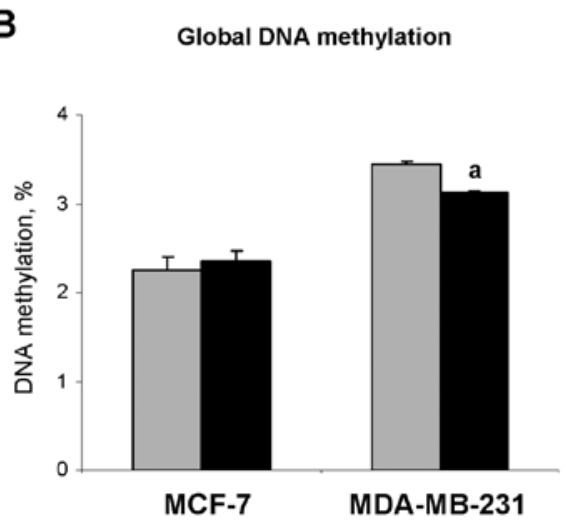

MDA-MB-231
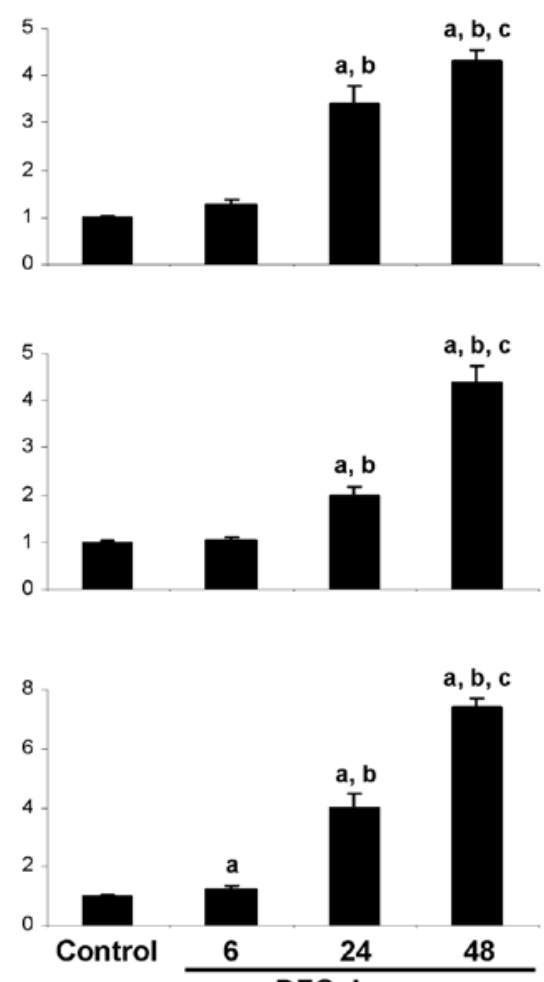

C

DFO, hours

CpG island methylation

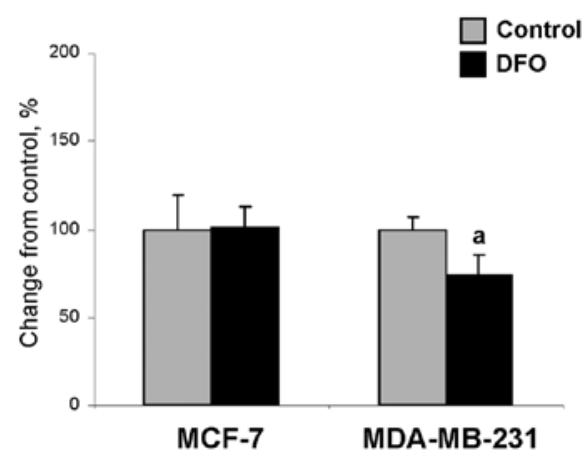

Figure 4. Effects of DFO treatment on the expression of the DNA methyltransferases, DNMT1, DNMT3A and DNMT3B, and DNA methylation in the MCF-7 and MDA-MB-231 human breast cancer cells. (A) The expression of DNMT1, DNMT3A and DNMT3B genes was determined by qRT-PCR as detailed in 'Materials and methods'. Data are presented as an average fold change in the expression of each gene in the DFO-treated cells relative to that in the corresponding untreated control cells, which were assigned the value 1 . The letters ' $a$ ', ' $b$ ' and ' $c$ ' denote a significant $(\mathrm{p}<0.05)$ difference $(\mathrm{n}=3)$ compared to the corresponding untreated control cells (a), or the cells treated with DFO for 6 (b) or $24 \mathrm{~h}$ (c). (B) Global DNA methylation in the untreated control and DFO-treated cells was measured using the LC-MS/MS method as detailed in 'Materials and methods'. Data are presented as a percentage of DNA methylation. The letter 'a' denotes a significant $(\mathrm{p}<0.05)$ difference $(\mathrm{n}=3)$ compared to the corresponding untreated control cells. $(\mathrm{C}) \mathrm{CpG}$ island methylation in the untreated control and DFO-treated cells was measured by $\left[{ }^{3} \mathrm{H}\right] \mathrm{dCTP}$ extension assay after digestion of DNA with methylation-sensitive $T s p$ MI restriction endonuclease. The $T s p$ MI hexanucleotide recognition sequences, CCCGGG, occur predominantly within CpG islands. Data are presented as a percentage change compared to the corresponding untreated control cells, which were assigned a value of $100 \%$. The letter ' $a$ ' denotes a significant $(\mathrm{p}<0.05)$ difference $(\mathrm{n}=3)$ compared to the corresponding untreated control cells.

$10 \%$. The level of $5-\mathrm{mC}$ in the $\mathrm{CpG}$ islands in the DFO-treated MCF-7 cells did not differ from that in the untreated MCF-7 control cells, whereas in the DFO-treated MDA-MB-231 cells, it was significantly decreased (Fig. 4C).

DFO affects the level of $p 53$ and $p 21$ proteins. Previous studies have indicated that the anti-tumor action of DFO and other iron chelators is associated with the activation of apoptosis (25-28); however, the mechanism underlying the induction of apoptosis has remained unexplored. Fig. 5 demonstrates that the treatment of wild-type TP53 MCF-7 cells with DFO resulted in an increase in the level of p53 protein in a time-dependent manner. The level of p53 protein in the DFO-treated cells at 6,24 and $48 \mathrm{~h}$ was 160, 211 and $370 \%$ greater, respectively, compared to the untreated MCF-7 cells. By contrast, an opposite trend in p53 alterations was observed in the DFO-treated mutant TP53 MDA-MB-231 cells. The treatment of MDA-MB-231 cells with DFO caused a progressive increase in $\mathrm{p} 21$ protein 

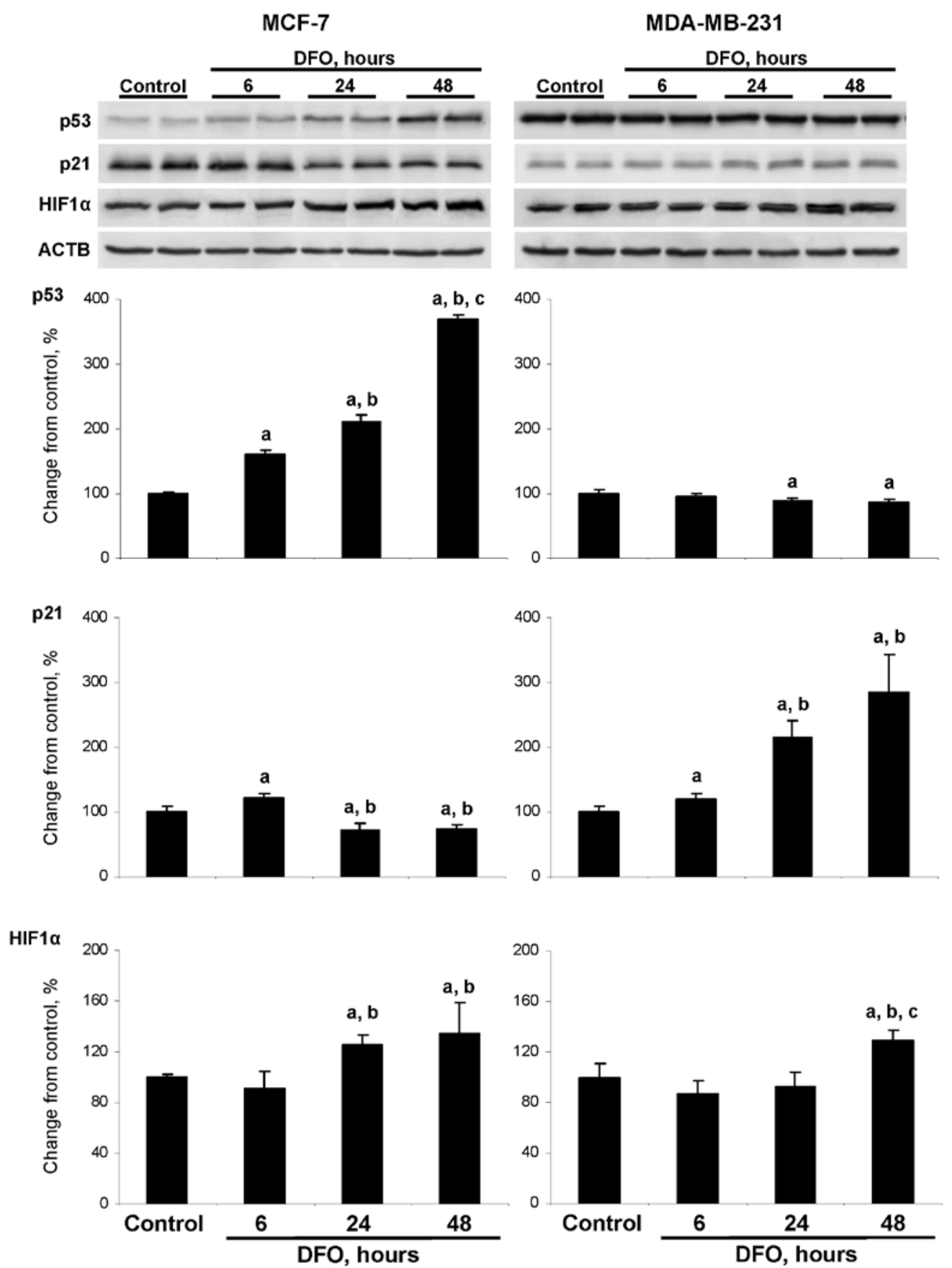

Figure 5. Effects of DFO treatment on the level sof p53, p21 and HIF1 $\alpha$ proteins in the untreated and DFO-treated MCF-7 and MDA-MB-231 human breast cancer cells. Cell lysates were separated by SDS-PAGE and subjected to western immunoblot analysis using specific antibodies against p53, p21 and HIF1 $\alpha$ proteins. Equal sample loading was confirmed by immunostaining against $\beta$-actin (ACTB). Representative western immunoblot images are shown. Data are presented as a percentage change compared to the corresponding untreated control cells, which were assigned a value of $100 \%$. The letters ' $a$ ', 'b' and 'c' denote a significant $(\mathrm{p}<0.05)$ difference $(\mathrm{n}=3)$ compared to the corresponding untreated control cells $(\mathrm{a})$, or the cells treated with DFO for 6 (b) or $24 \mathrm{~h}(\mathrm{c})$.

levels, with the highest values being detected at 24 and $48 \mathrm{~h}$, while the levels of p21 protein in the DFO-treated MCF-7 cells decreased. The treatment of MCF-7 and MDA-MBA-231 cells with DFO caused a moderate elevation in HIF1 $\alpha$ protein levels with changes being significant at 24 an $48 \mathrm{~h}$ in the MCF-7 cells and at $48 \mathrm{~h}$ in the MDA-MB-231 cells.

DFO affects the histone methylation pattern at the p21 gene promoter. Previous reports have suggested a link between the induction of apoptosis and an epigenetic mechanism for the transcriptional activation of the $p 21$ gene $(22,29)$. In view of this, and considering the substantial global epigenetic changes induced by DFO, we used ChIP assay to examine the levels of histones H3K9me2 and H3K4me2, 2 epigenetic markers associated with the alteration of gene expression, at the promoter of the $p 21$ gene. Fig. 6 demonstrates that at $48 \mathrm{~h}$ after treatment, the DFO-treated MCF-7 cells were characterized by a prominent increase in the levels of histones $\mathrm{H} 3 \mathrm{~K} 9 \mathrm{me} 2$ and $\mathrm{H} 3 \mathrm{~K} 4 \mathrm{me} 2$. At this time point, the levels of histones H3K9me2 and $\mathrm{H} 3 \mathrm{~K} 4 \mathrm{me} 2$ were 12.3- and 7.1-fold greater, respectively, 

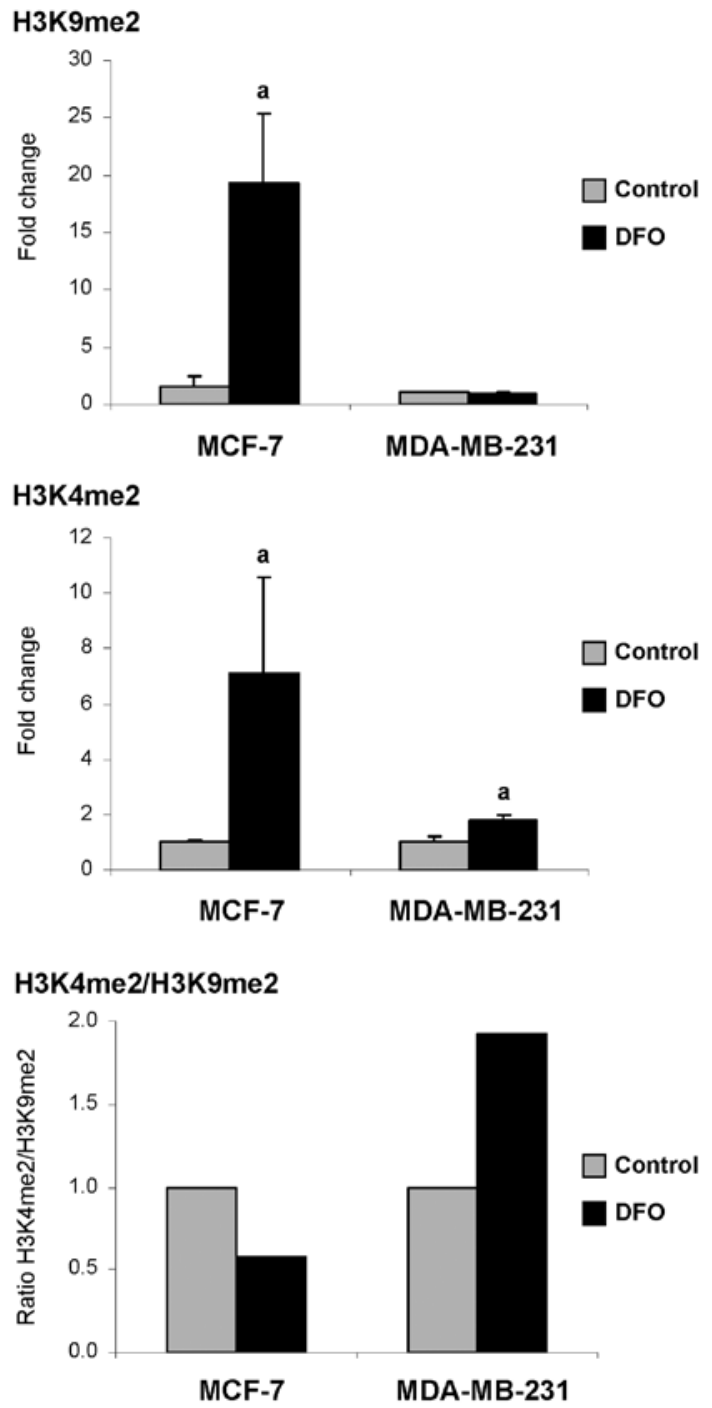

Figure 6. Effects of DFO treatment on the level of histone H3 lysine methylation at the $p 21$ gene promoter in the MCF-7 and MDA-MB-231 human breast cancer cells. ChIP assay was performed with primary antibodies against dimethyl-histone $\mathrm{H} 3$ lysine 9 and dimethyl-histone $\mathrm{H} 3$ lysine 4. Purified DNA from the enriched fragments and from input DNA was amplified by qPCR with $p 21$-specific primer sets. The data are presented as a fold change relative to the corresponding control untreated cells after normalization to input DNA. The letter ' $a$ ' denotes a significant $(\mathrm{p}<0.05)$ difference $(n=3)$ compared to the corresponding untreated control cells.

compared to the untreated control cells. By comparison, the DFO-treated MDA-MB-231 cells exhibited a 1.8-fold increase in the levels of histone $\mathrm{H} 3 \mathrm{~K} 4 \mathrm{me} 2$, while the levels of histone H3K9me2 did not differ from the control values. More importantly, the $\mathrm{H} 3 \mathrm{~K} 4 \mathrm{me} 2 / \mathrm{H} 3 \mathrm{~K} 9 \mathrm{me} 2$ ratio in the DFO-treated MDA-MB-231 cells was 1.9 -fold greater compared to the control cells, indicating an enrichment of the $p 21$ promoter by histone H3K4me2. By contrast, in the DFO-treated MCF-7 cells, the H3K4me2/H3K9me2 ratio was decreased, indicating an enrichment of the $p 21$ gene promoter by the transcriptional silencing of histone $\mathrm{H} 3 \mathrm{~K} 9 \mathrm{me} 2$.

DFO enhances the sensitivity of MCF-7 and MDA-MB-231 cancer cells to chemotherapeutic drugs. Given that DFO treatment induced pro-apoptotic pathways in the MCF-7 and MDA-MB-231 cells, we investigated whether the activation
Table I. Drug sensitivity of MCF-7 and MDA-MB-231 cells following treatment with desferrioxamine (DFO).

\begin{tabular}{|c|c|c|c|c|}
\hline \multirow[b]{2}{*}{ Drug } & \multicolumn{2}{|c|}{ MCF-7 } & \multicolumn{2}{|c|}{ MDA-MB-231 } \\
\hline & Control & DFO & Control & DFO \\
\hline DOX & $4.4 \pm 0.2$ & $3.7 \pm 0.2$ & $9.2 \pm 0.2$ & $1.7 \pm 0.1^{\mathrm{a}}$ \\
\hline CDDP & $25.9 \pm 3.7$ & $14 \pm 2.9^{\mathrm{a}}$ & $38.9 \pm 8.6$ & $23.6 \pm 5.2^{\mathrm{a}}$ \\
\hline
\end{tabular}

Data are presented as an average of the $\mathrm{IC}_{50}$ values. ${ }^{\text {a Significant }}(\mathrm{p}<0.05)$ difference compared to the corresponding untreated control cells $(n=3)$. DOX, doxorubicin hydrochloride; CDDP, cis-diammineplatinum(II) dichloride.

of these pathways results in increased cancer cell sensitivity to chemotherapeutic drugs. Table I demonstrates that in the DFO-treated MCF-7 and MDA-MB-231 cells, the $\mathrm{IC}_{50}$ values for DOX and CDDP were lower than the $\mathrm{IC}_{50}$ values in the untreated cells. The most dramatic increase in drug sensitivity occurred with the MDA-MB-231 cells cultured in DFO-containing medium and treated with DOX.

\section{Discussion}

Breast cancer is one of the most prevalent malignancies in women (30). Despite the statistically significant decline in breast cancer incidence in recent years, breast cancer is currently the leading cause of cancer-related mortality among women worldwide (31) and second leading cause of cancer-related mortality among women in the US (30). The success of breast cancer treatment relies on a better understanding of the underlying molecular mechanisms involved in breast cancer initiation and progression. Therefore, further the comprehensive elucidation of breast cancer-associated molecular abnormalities is critical in order to improve the clinical management of breast cancer.

In recent years, the role and mechanisms of breast cancerrelated abnormalities in iron metabolism have been investigated in a variety of experimental and clinical settings (11,32-34), with particular emphasis on altering the dependence of cancer cells on iron as a potential therapeutic strategy for the treatment of tumors and/or increasing the efficacy of anti-cancer agents $(17,35,36)$. This strategy is driven largely by the well-established fact that neoplastic cells have an increased requirement for iron, as well as recent evidence demonstrating that 'high intracellular iron phenotype' is associated with poor prognosis in breast cancer patients (33). Several potential mechanisms have been proposed for the anti-cancer effects of iron chelating compounds, including the inhibition of iron-dependent ribonucleotide reductase (15), a rate-limiting enzyme in DNA synthesis, the inhibition of cell cycle progression by inducing $\mathrm{G}_{1}-\mathrm{S}$ phase arrest (15), and the inhibition of epithelial-to-mesenchymal transition via the up-regulation of N-Myc downstream-regulated gene 1 (NDRG1) (37).

Iron is a key regulator of cellular metabolic reactions, including several DNA and histone-modifying proteins $(7,8)$. In the present study, we demonstrate that the iron chelator, DFO, substantially decreases the protein levels of the histone H3K9 demethylase, JMJD2A, in MCF-7 and MDA-MB-231 breast 
cancer cells (Fig. 1). The reduction in JMJD2A levels following DFO treatment was not a surprising discovery, since JMJD2A belongs to the superfamily of iron-dependent oxygenases (11). This suggests that the down-regulation of JMJD2A is associated directly with the iron chelating properties of DFO; however, in the DFO-treated MCF-7 cells, HIF1 $\alpha$ may also actively contribute to this effect (38), since it was moderately up-regulated (Fig. 5).

DFO treatment also caused a down-regulation of the histone H3K4 demethylase, LSD1, in the MDA-MB-231 cells (Fig. 1). This was unexpected since LSD1 belongs to the family of flavin-dependent amine oxidases (39); however, it has been recently demonstrated that LSD1 exhibits folate-binding activity (40). Considering the intimate interdependence between iron, flavin and folate metabolic pathways, alterations in intracellular iron metabolism may indirectly affect the level and function of LSD1.

The down-regulation of JMJD2A and LSD1 in the DFOtreated cells was accompanied by the altered expression of a number of histone $\mathrm{H} 3 \mathrm{~K} 9, \mathrm{H} 3 \mathrm{~K} 36$ and $\mathrm{H} 3 \mathrm{~K} 4$ methyltransferase genes (Fig. 3). These down- and up-regulation events were accompanied by marked changes in the global levels of corresponding metabolically sensitive histone markers. Specifically, the DFO-treated MCF-7 cells exhibited a prominent increase in the levels of H3K9me2 and H3K36me3, while the DFO-treated MDA-MB-231 cells were characterized by an increase in the levels of H3K4me2 (Fig. 6). The differential expression levels of H3K9me2 and H3K4me2 in the DFO-treated MCF-7 and MDA-MB-231 cells were associated with the differential expression patterns of histone demethylases and HMTs in these cells.

Previous reports have shown that the treatment of various cancer cells with DFO induces apoptosis through the activation of various pro-apoptotic pathways (25-28). Likewise, the results of the present study demonstrated that the exposure of MCF-7 and MDA-MB-231 human breast cancer cells to DFO induced the pro-apoptotic program; however, the underlying mechanisms of this activation differed in these cell lines. The treatment of MCF-7 cells that possess wild-type TP53 with DFO activated a p53-dependent pro-apoptotic pathway, which was evident by the profound increase in p53 protein levels in the MCF-7treated cells (Fig. 5). It has been suggested that the JMJD2A histone demethylase plays a major role in the regulation of apoptosis. Specifically, several studies have demonstrated that the depletion of JMJD2A increases p53-dependent apoptosis (41-43). The findings of the present study showing a substantial reduction in JMJD2A protein expression concomitant with an increase in p53 protein levels in the DFO-treated MCF-7 cells are in accord with this suggestion.

By contrast, the treatment of mutant TP53 MDA-MB-231 cells with DFO also activated a pro-apoptotic program, although in a p53-independent manner, as evident by the substantial increase in p21 protein levels. Several possible explanations exist for the mechanism of p21-up-regulation in the DFO-treated MDA-MB-231 cells. First, it may be attributed to the enrichment of the promoter region of the $p 21$ gene by $\mathrm{H} 3 \mathrm{~K} 4 \mathrm{me} 2$, a histone modification marker that is strongly associated with active transcription. This was evident by the more extensive increase in histone $\mathrm{H} 3 \mathrm{~K} 4 \mathrm{me} 2$ levels compared to histone $\mathrm{H} 3 \mathrm{~K} 9 \mathrm{me} 2$ levels at the promoter region of the p21 gene, which corresponds to previous findings showing that the methylation of histone H3K4 impairs the methy- lation of histone $\mathrm{H} 3 \mathrm{~K} 9$ (44). By contrast, in the DFO-treated MCF-7 cells with down-regulated $p 21$, the promoter region of the p21 gene was enriched by the transcriptional silencing of $\mathrm{H} 3 \mathrm{~K} 9 \mathrm{me} 2$. Second, it has been demonstrated that the histone demethylase, JMJD2A, can bind to the promoter of the $p 21$ gene and repress its expression (43). Therefore, the DFO-induced decrease in JMJD2A protein expression may block its recruitment to the $p 21$ gene with a resultant enhancement in the expression of $p 21$. Third, recent evidence has indicated that a DFO-induced up-regulation of the NDRG1 gene (37) may up-regulate p21 expression via p53-independent mechanisms (45).

The results of this study also show that the activation of apoptotic pathways in the DFO-treated breast cancer cells enhances the anti-cancer activity of chemotherapeutic drugs. The most pronounced changes in drug-sensitivity were the increased sensitivity of MDA-MB-231 cells, which possess an advanced mesenchymal and drug-resistant phenotype (46), to DOX, one of the main chemotherapeutic agents for the treatment of breast cancer. In addition to the up-regulation of the p53-independent pro-apoptotic pathway, this may be attributed to the activation of other epigenetically silenced tumor suppressor genes in the MDA-MB-231 cells, which was evident by a decrease in the 5-mC levels.

In conclusion, to our knowledge, the results of the present study provide evidence for the first time that the iron chelator, DFO, activates apoptotic programs in human breast cancer cells and enhances their sensitivity to chemotherapeutic agents via epigenetic mechanisms by affecting the functioning of the chromatin remodeling machinery. The activation of the pro-apoptotic program in wild-type TP53 MCF-7 cells was p53-dependent, triggered mainly by the down-regulation of the JMJD2A histone demethylase. In mutant TP53 MDA-MB-231 cells, DFO caused similar effects via the activation of the p53-independent apoptotic program driven predominantly by the epigenetic up-regulation of p21. Importantly, the results of the present study provide experimental proof of the interdependence between iron and epigenetic regulatory mechanisms and suggest that the modification of intracelluar iron metabolism may enhance the efficacy of epigenetic therapy.

\section{Acknowledgements}

The views expressed in this study do not necessarily represent those of the US Food and Drug Administration.

\section{References}

1. Teperino R, Schoonjans K and Auwerx J: Histone methyltransferases and demethylases; can they link metabolism and transcription? Cell Metab 12: 321-327, 2010.

2. Burgio G, Onorati MC and Corona DFV: Chromatin remodeling regulation by small molecules and metabolites. Biochim Biophys Acta 1799: 671-680, 2010.

3. Hou $\mathrm{H}$ and $\mathrm{Yu} \mathrm{H}$ : Structural insights into histone lysine demethylation. Curr Opin Struct Biol 20: 739-748, 2010.

4. Iyer LM, Abhiman S and Aravind L: Natural history of eukaryotic DNA methylation systems. Prog Mol Biol Transl Sci 101: 25-104, 2011.

5. Tsukada Y-i, Fang J, Erdjument-Bromage H, Warren ME, Borchers $\mathrm{CH}$, Tempst $\mathrm{P}$ and Zhang Y: Histone demethylation by a family of JmjC domain-containing proteins. Nature 439: 811-816, 2006. 
6. Yamane K, Toumazou C, Tsukada Y-i, Erdjument-Bromage H, Tempst $\mathrm{P}$, Wong J and Zhang Y: JHDM2A, a JmjC-containing H3K9 demethylase, facilitates transcription activation by androgen receptor. Cell 125: 483-495, 2006.

7. Wu $\mathrm{H}$ and Zhang Y: Mechanisms and functions of Tet proteinmediated 5-methylcytosine oxidation. Genes Dev 25: 2436-2452, 2011.

8. McDonough MA, Loenarz C, Chowdhury R, Clifton IJ and Schofield CJ: Structural studies of human 2-oxoglutarate dependent oxygenases. Curr Opin Struct Biol 20: 659-672, 2010.

9. Torti SV and Torti FM: Ironing out cancer. Cancer Res 71: 1511-1514, 2011

10. Jones PA, Baylin SB: The epigenomics of cancer. Cell 128: 683-692, 2007.

11. Miller LD, Coffman LG, Chou JW, Black MA, Berg J, D'Agostino R Jr, Torti SV and Torti FM: An iron regulatory gene signature predicts outcome in breast cancer. Cancer Res 71 : 6728-6737, 2011

12. Hanahan D and Weinberg RA: Hallmarks of cancer: the next generation. Cell 144: 646-674, 2011.

13. Baylin SB and Jones PA: A decade of exploring the cancer epigenome - biological and translational implications. Nat Rev Cancer 11: 726-734, 2011

14. Buss JL, Torti FM and Torti SV: The role of iron chelation in cancer therapy. Curr Med Chem 10: 1021-1034, 2003.

15. Yu Y, Gutierrez E, Kovacevic Z, Saletta F, Obeidy P, Suryo Rahmanto Y and Richardson DR: Iron chelators for the treatment of cancer. Curr Med Chem 19: 2689-2702, 2012.

16. Kawamoto M, Horibe T, Kohno M and Kawakami K: A novel transferring receptor-targeted hybrid peptide disintegrates cancer cell membrane to induce rapid killing of cancer cells. BMC Cancer 11: 359, 2011.

17. Rao VA, Klein SR, Agama KK, Toyoda E, Adachi N, Pommier Y, and Shacter EB: The iron chelator Dp44mT causes DNA damage and selective inhibition of topoisomerase II $\alpha$ in breast cancer cells. Cancer Res 69: 948-957, 2009.

18. Chekhun VF, Lukyanova NY, Kovalchuk O, Tryndyak VP and Pogribny IP: Epigenetic profiling of multidrug-resistant MCF-7 breast cancer adenocarcinoma cells reveals novel hyper- and hypomethylated targets. Mol Cancer Ther 6: 10891098, 2007.

19. Schmittgen TD and Livak KJ: Analyzing real-time PCR data by the comparative CT method. Nat Protoc 3: 1101-1108, 2008.

20. Zhang JJ, Zhang L, Zhou K, Ye X, Liu C, Zhang L, Kang J and Cai C: Analysis of global DNA methylation by hydrophilic interaction ultra high-pressure liquid chromatography tandem mass spectrometry. Anal Biochem 413: 164-170, 2011.

21. Pogribny I, Yi P and James SJ: A sensitive new method for rapid detection of abnormal methylation patterns in global DNA and within $\mathrm{CpG}$ islands. Biochem Biophys Res Commun 262: 624-628, 1999

22. Kim TD, Shin S, Berry WL, Oh S and Janknecht R: The JMJDA demethylase regulates apoptosis and proliferation in colon cancer cells. J Cell Biochem 113: 1368-1376, 2012

23. Stewart MD, Li J and Wong J: Relationship between histone H3 lysine 9 methylation, transcription repression, and heterochromatin protein 1 recruitment. Mol Cell Biol 25: 2525-2538, 2005.

24. Shi Y, Lan F, Matson C, Mulligan P, Whetstine JR, Cole PA, Casero RA and Shi Y: Histone demethylation mediated by the nuclear amine oxidase homolog LSD1. Cell 119: 941-953, 2004.

25. Hileti D, Panayiotidis $\mathrm{P}$ and Hoffbrand AV: Iron chelators induce apoptosis in proliferating cells. Br J Haematol 89: 181-187, 1995.

26. Pan YJ, Hopkins RG and Loo G: Increased GADD153 gene expression during iron chelation-induced apoptosis in Jurkat T-lymphocytes. Biochim Biophys Acta 1691: 41-50, 2004.

27. So EY, Ausman M, Saeki T and Ouchi T: Phosphorylation of SMC1 by ATR is required for desferrioxamine (DFO)-induced apoptosis. Cell Death Dis 2: e128, 2011.
28. Saletta F, Suryo Rahmanto Y, Siafakas AR and Richardson DR Cellular iron depletion and the mechanisms involved in the iron-dependent regulation of the growth arrest and DNA damage family of genes. J Biol Chem 286: 35396-35406, 2011.

29. Escoubet-Lozach L, Lin IL, Jensen-Pergakes K, Brady HA Gandhi AK, Schafer PH, Muller GW, Worland PJ, Chan KW and Verhelle D: Pomalidomide and lenalidomide induce p21WAF-1 expression in both lymphoma and multiple myeloma through a LSD1-mediated epigenetic mechanism. Cancer Res 69: 7347-7356, 2009.

30. Siegel R, Naishadham D and Jemal A: Cancer statistics, 2012. CA Cancer J Clin 62: 10-29, 2012.

31. Jemal A, Bray F, Center MM, Ferlay J, Ward E and Forman D: Global cancer statistics. CA Cancer J Clin 61: 69-90, 2011.

32. Huang X: Does iron have a role in breast cancer? Lancet Oncol 9 803-807, 2008

33. Pinnix ZK, Miller LD, Wang W, D'Agostino R Jr, Kute T, Willngham MC, Hatcher H, Tesfay L, Sui G, Di X, Torti SV and Torti FM: Ferroportin and iron regulation in breast cancer progression and prognosis. Sci Transl Med 2: 43ra56, 2010.

34. Shpyleva SI, Tryndyak VP, Kovalchuk O, Starlard-Davenport A, Chekhun VF, Beland FA and Pogribny IP: Role of ferritin alterations in human breast cancer cells. Breast Cancer Res Treat 126: 63-71, 2011

35. Hoke EM, Maylock CA and Shacter E: Desferal inhibits breast tumor growth and does not interfere with the tumoricidal activity of doxorubicin. Free Radic Biol Med 39: 403-411, 2005.

36. Whitnall M, Howard J, Ponka P, and Richardson DR: A class of iron chelators with a wide spectrum of potent antitumor activity that overcomes resistance to chemotherapeutics. Proc Natl Acad Sci USA 103: 14901-14906, 2006.

37. Chen Z, Zhang D, Yue F, Zheng M, Kovacevic Z and Richardson DR: The iron chelators Dp44mT and DFO inhibit TGF- $\beta$-induced epithelial-mesenchymal transition via up-regulation of N-Myc downstream-regulated gene-1 (NDRG1). J Biol Chem 287: 17016-17028, 2012.

38. Pollard PJ, Loenarz C, Mole DR, McDonough MA, Gleadle JM, Schofield CJ and Ratcliffe PJ: Regulation of Jumonji-domaincontaining histone demethylases by hypoxia-inducible factor (HIF)-1 $\alpha$. Biochem J 416: 387-394, 2008.

39. Culhane JC and Cole PA: LSD1 and the chemistry of histone demethylation. Curr Opin Chem Biol 11: 561-568, 2007.

40. Luka Z, Moss F, Loukachevitch LV, Bornhop DJ and Wagner C: Histone demethylase LSD1 is a folate-binding protein. Biochemistry 50: 4750-4756, 2011

41. Black JC, Allen A, Van Rechem C, Forbes E, Longworth M, Tschöp K, Rinehart C, Quiton J, Walsh R, Smallwood A, Dyson NJ and Whetstine JR: Conserved antagonism between JMJD2A/KDM4A and HP1 $\gamma$ during cell cycle progression. Mol Cell 40: 736-748, 2010.

42. Mallette FA, Mattiroli F, Cui G, Young LC, Hendzel MJ, Mer G, Sixma TK and Richard S: RNF8- and RNF168-dependent degradation of KDM4A/JMJD2A triggers 53BP1 recruitment to DNA damage sites. EMBO J 31: 1865-1878, 2012.

43. Kim TD, Oh S, Shin S and Janknecht R: Regulation of tumor suppressor p53 and HCT116 cell physiology by histone demethylase JMJD2D/KDM4D. PLoS One 7: e34618, 2012.

44. Binda O, LeRoy G, Bua DJ, Garcia BA, Gozani O and Richard S: Trimethylation of histone $\mathrm{H} 3$ lysine 4 impairs methylation of histone $\mathrm{H} 3$ lysine 9. Regulation of lysine methyltransferases by physical interaction with their substrates. Epigenetics 5: 767-775, 2010.

45. Kovacevic Z, Sivagurunathan S, Mangs H, Chikhani S, Zhang D and Richardson DR: The metastasis suppressor, $\mathrm{N}$-myc downstream regulated gene 1 (NDRG1), upregulates $\mathrm{p} 21$ via p53-independent mechanisms. Carcinogenesis 32: 732-740, 2011.

46. Tryndyak VP, Beland FA and Pogribny IP: E-cadherin transcriptional down-regulation by epigenetic and microRNA-200 family alterations is related to mesenchymal and drug-resistant phenotypes in human breast cancer cells. Int J Cancer 126: 2575-2583, 2010 